\title{
Artificial Neural Networks on Recruiting Athletes
}

\section{Tales Scopinho}

\section{Centennial Collegiate Vocational Institute}

Humanity's relationship with sports goes back as far as 1700 BCE with records of boxing fights on the Akrotiri fresco. The Greeks have long been fascinated with sporting competitions as their first Olympics dates back to $776 \mathrm{BC}$. Athletic events like these revealed the ingrained human desire to win competitions and witness the exoneration of the human physique since the dawn of our civilization. An important aspect of achieving success in team sports is the ability to optimally construct a winning team through a careful selection and combination of athletes. In the Ancient Olympic Games, cities recruited athletes from other villages to demonstrate national prestige [1]. In addition, the training period before the Games ensured high athletic standards the length of the training period without any financial reward deterred less affluent athletes who had little hope of success from participating [1].

Nowadays, major sporting leagues are aware of the extent to which athlete recruitment contributes to the league's playing level, and therefore have developed methods to assist in athlete recruitment. The largest change yet has been the introduction of the draft, which provides teams with an opportunity to select promising young athletes. Originally, coaches and scouts relied solely on their vision and intuition to decide whether to pick a certain player in the draft, but in modern times, advancements in data analytics and statistics have dramatically changed the methods that teams use to identify and recruit athletes [2]. In major sports leagues, every organization has a team of highly capable personnel in charge of accumulating datasets on teams and individual players. Furthermore, the rise of Artificial Intelligence being utilized in data analysis introduced a revolutionary alternative Artificial Neural Networks (ANNs) - to analyzing performance data and assisting teams' decisions. In this viewpoint, it will be argued that the characteristics of ANNs make it a reliable tool for player recruitment that should be further developed and implemented in sports analytics.

ANNs are a method of data analysis that was developed based on the analogy of neuronal connections in the human brain. The complex connections in the brain give humans the ability to learn from previous experiences. These neural connections inspired the architecture of ANNs, which have the ultimate goal of learning the best weighting of evidence to predict an outcome, based on real-life examples. Nonetheless, neural networks also feature algorithms with no cognitive plausibility, like backpropagation. The training of the network occurs by feeding it data that contains multiple examples of inputs matched with their expected behaviour. From this information, the network detects correlations between the inputs and the desired outputs and adjusts its variables through backpropagation to consistently achieve the expected behaviour. After the training period is complete, new data is utilized for testing [3]. The human brain is a complex organ that requires a back-end system of an estimated $10^{11}$ (100 billion) [4] neurons of various lengths and types to work [5]. Networks of these biological neurons are the functioning units of the brain, and early neural networks attempted to recreate concepts from neuroscience, like the "all-or-none response", with artificial neurons.

Artificial neurons are a simplified model of brain neurons. Similar to brain neurons, an ANN is also comprised of a series of neurons with similar features connected to each other. An ANN, more specifically a Multi-Layer Perceptron (MLP) network, is made up of three main interconnected layers: an Input Layer, Hidden Layers and an Output Layer [6], as observed in Figure 2.

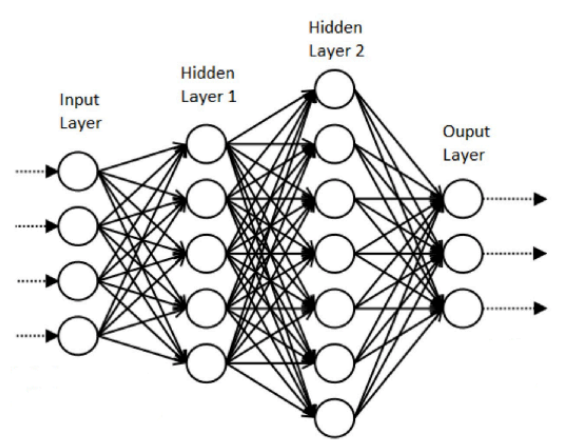

Figure 2. The General Structure of a Multi-Layer Perceptron Neural Network. Each line indicates a signal path. (source: shorturl.at/OPUX5) 
The learning process of the model starts in the Input Layer where every node is clamped to a numerical value, which is then forwarded to the Hidden Layers. Each neuron in the Hidden Layers, referred to as a perceptron, performs individual calculations (further explained in this paper). This processing may be simple (summing the inputs), or complex (a node might contain another network) [7]. After the process is complete, the results are then transmitted to the next higher layer, culminating in the Output Layer.

Artificial Neurons receive a variety of numerical inputs (the number of inputs varies depending on the network) and output a single number, often range-bounded to between 0 and 1 [8]. Every input, $x$, to an artificial neuron has a single number called a weight, $w$, assigned to it. All inputs are multiplied by their individual weights before being added together [4][7], whereupon the neuron often functions similarly to a biological neuron's "all-or-none response." The artificial neuron's output is 0 (deactivated) if the weighted sum is less than or greater than a specific threshold value, and 1 (activated) if the value is greater than the threshold [8]. The algebraic output of a perceptron is shown in Figure 3.0.

$$
\text { output }=\left\{\begin{array}{lll}
0 & \text { if } & \sum_{j} w_{j} x_{j} \leq \text { threshold } \\
1 & \text { if } & \sum_{j} w_{j} x_{j}>\text { threshold }
\end{array}\right.
$$

Figure 3.0. Output of an artificial neuron

The output of a perceptron equation could be rearranged if we treat $\sum_{\mathrm{j}} w_{j} x_{j}$ as a dot product of two vectors where vectors $\mathrm{w}$ and $\mathrm{x}$ have, respectively, weights and inputs as components. In addition, if the threshold is moved to the other side of the equation and replaced with a bias $(b \equiv-$ threshold), the equation from Figure 3.0 becomes:

$$
\text { output }=\left\{\begin{array}{lll}
0 & \text { if } & w \cdot x+b \leq 0 \\
1 & \text { if } & w \cdot x+b>0
\end{array}\right.
$$

Figure 3.1. Rewritten output of an artificial neuron

The bias is a measure of how easy it is to get the neuron to fire, although most modern neural networks make use of sigmoidal neurons instead of perceptrons. Sigmoidal neurons follow the same concept, except their output is an analogic number between 0 and 1 . This number is achieved by the Sigmoid Function, $\sigma(z) \equiv \frac{1}{1+e^{-z}}, \quad$ that converts any value, $z$, to its equivalent in the interval of 0 and 1 . The output of the sigmoidal neuron is computed as $\sigma(z) \equiv \frac{1}{1+\exp \left(-\sum_{j} \omega_{j} x_{j}-b\right)}$

[8].
In Backpropagation Neural Networks, data containing inputs and the desired theoretical output is used to train the MLP. Initially, the network assigns random values to the weights and biases. For every subsequent round of training, the ANN performs the calculation with the inputs and compares the experimental output to the theoretical output. Based on the calculated deviation, the network runs backwards: it starts at the final layer and adjusts the weights and biases for every node [8]. This process is repeated for a number of training rounds, and after multiple runs, the ANN begins to identify patterns in the data.

ANNs have been vastly used in the sports field in many ways; they have been used to model performance and rank National College Athletic Association (NCAA) college football teams [10], identify patterns in National Basketball Association (NBA) games [11], predict winners of the National Football League (NFL) championship [12], and to analyze players' configurations in volleyball games [13]. The majority of machine learning research conducted in sports is focused on predicting outcomes of matches or finding patterns in statistics, but few studies have used ANNs to aid in athlete recruitment.

In existing research, the use of ANNs has proved to be an effective aid in the selection of players and performance prediction. Although the results reveal that ANNs cannot completely replace human selections, there is enough evidence to conclude that ANNs hold great potential as a tool for recruiters and scouts. In general, more advanced architectures including Ensemble Neural Networks and Radial Basis Function Networks outperformed other simpler models. However, there is no unique approach that was proven accurate for all cases since similar architectures were shown to vary in accuracy from case to case. The following three studies demonstrate the reliability of ANNs of various architectures to predict player recruitment across multiple sports:

1) McCullagh, "Data Mining in Sport: A Neural Network Approach." [14] In his research, McCullagh uses multiple backpropagation networks to rank U18 football players from the Australian Football League (AFL) Draft Camp and predict their future playing ability. The author developed a Modular Neural Network: a neural network that decomposes the problem into sub-tasks that are tackled by separate models. The network inputs attributes of a player including anthropometric, psychological and skill assessment; and 
outputs a rating from 0 to 10 . The study performed three different experiments, comparing the results with Recruiting Managers (RMs) from all 16 AFL clubs to test for accuracy. In all trials, the ANNs did not outperform the selections made by the managers alone, although the results only differed by $5.5 \%$ in the best case. However, when RM and ANNs agreed on the same classification, the predictions had their accuracy increased by as much as $5.7 \%$ compared to when RMs were correct alone. In conclusion, in this study, the ANNs were successful as "an additional source of information to assist in the decision-making process." [14]

2) lyer, Sharda, "Prediction of athletes performance using neural networks: An application in cricket team selection." [15] In their paper, lyer and Sharda applied neural networks to aid in selecting cricket players for international competitions. In brief, the researchers performed one experiment that compared results from 5 different ANNs in player selections, and another that considered the nationality of the athletes and created 10 ANNs for each country competing in the Cricket World Cup. The results showed that in all cases, the networks clearly outperformed the selections from the selection committee, granting a value addition "of $35 \%$ in experiment 1 and 17\% in experiment 2." [15]

3) Edelmann-nusser, Hohmann, Henneberg, “Modeling and prediction of competitive performance in swimming upon neural networks." [16] In individual sports, such as competitive swimming, athlete recruitment is mostly done based on predicting their individual performances. The authors gathered data consisted of 19 performances of female swimmers in the $200-\mathrm{m}$ backstroke category as well as practice data to predict their competitive performance at the Olympic Games. The paper primarily focused on the impacts of different training strategies taken by athletes preceding competitions; however, the results also show the accuracy of MLPs in predicting individual sports performances. The model was trained to predict the final time of athletes in 200-m backstrokes. After the training period, the MLP had a mean error of $+/-0.62 \mathrm{~s}$. As a comparison, the mean time of all nineteen 200-m backstroke races was 2:12.94 min:s. In conclusion, the overall model predicted an Olympic performance of 2:12.59 min:s, while the real competitive performance was 2:12.64 min:s (an error of $0.05 \mathrm{~s}$ or $1 / 20$ of a second).

ANNs, more specifically Multi-Layer Perceptron Networks, are reliable in predicting athlete performances in individual sports given the proper training data. On team sports, ANNs were proven to be effective in assisting the selection of athletes to sports teams, though they did not always outperform human selection. This was expected given that neural networks rely solely on the data provided to do their analysis, whereas real selection committees have data including "numerous subjective opinions on skills and fitness, performance in key games, family background, [and] improvement over time" [14]. In addition, team sports require a conjoint effort to win as all the pieces must collaborate and fit together to be successful. None of the research accounted for the chemistry of the selected team nor for how the players selected would fit and play together on a team. These facts alone are some of the most challenging aspects to account for in a neural network due to the aggregate type of the information. Also, the problem becomes even more challenging because the players are on an as-yet non-existent team with very incomplete previous data to analyze player combinations and team statistics. As the research stands, there are limitations to using ANNs for player recruitment in specific scenarios. However, ANNs and player recruitment are an extremely new pairing in the world of sports analytics, and it is only in the early stages of development. Further research and development need to be conducted using realworld scenarios to improve the breadth of ANNs' analytical usefulness and establish it as a leading method for player recruitment.

\section{ACKNOWLEDGEMENTS}

I hereby acknowledge the sponsorship of the STEM Fellowship Program, as well as Tristan Wild for assisting with the manuscript editing.

\section{REFERENCES}

1. Crowther NB. Athlete and state: qualifying for the Olympic Games in ancient Greece. Journal of Sport History. 1996 Apr 1;23(1):34-43.

2. Berri DJ, Brook SL, Fenn AJ. From college to the pros: predicting the NBA amateur player draft. Journal of Productivity Analysis. 2010;35(1):25-35.

3. Bartlett R. Artificial intelligence in sports biomechanics: New dawn or false hope?. Journal of sports science \& medicine. 2006 Dec;5(4):474. 
4. Gurney K. An introduction to neural networks. CRC press; 2014 Apr 21.

5. Schalkoff RJ. Artificial neural networks. McGraw-Hill Higher Education; 1997 Jun 1.

6. Krishnagopal Dharani. Chapter 2 - Physiology of the Neuron, The Biology of Thought. Academic Press. 2015. doi: 10.1016/B978-0-12-800900-0.00002-6.

7. Gershenson C. Artificial neural networks for beginners. arXiv preprint cs/0308031. 2003 Aug 20.

8. Nielsen MA. Neural networks and deep learning. San Francisco, CA, USA:: Determination press; 2015 Sep 25.

9. Basheer IA, Hajmeer M. Artificial neural networks: fundamentals, computing, design, and application. Journal of microbiological methods. 2000 Dec 1;43(1):3-1.

10. Wilson RL. Ranking college football teams: A neural network approach. Interfaces. 1995 Aug;25(4):44-59.

11. Bhandari I, Colet E, Parker J, Pines Z, Pratap R, Ramanujam K. Advanced scout: Data mining and knowledge discovery in NBA data. Data Mining and Knowledge Discovery. 1997 Mar 1;1(1):121-5.

12. Kahn J. Neural network prediction of NFL football games. World Wide Web electronic publication. 2003 Dec 19:9-15.

13. Jäger JM, Perl J, Schöllhorn IW. Analysis of players' configurations by means of artificial neural networks. International Journal of Performance Analysis in Sport. 2007 Oct 1;7(3):90-105.

14. McCullagh J. Data mining in sport: a neural network approach. International Journal of Sports Science and Engineering. 2010;4(3):131-8.

15. Iyer SR, Sharda R. Prediction of athletes performance using neural networks: An application in cricket team selection. Expert Systems with Applications. 2009 Apr 1;36(3):5510-22.

16. Edelmann-Nusser J, Hohmann A, Henneberg B. Modeling and prediction of competitive performance in swimming upon neural networks. European Journal of Sport Science. 2002 Apr 1;2(2):1-0. 


\section{Conference Proceedings}

